\title{
Differential effects of muscarinic receptor blockade in prelimbic cortex on acquisition and memory formation of an odor-reward task
}

\author{
Anna Carballo-Márquez, Anna Vale-Martínez, ${ }^{1}$ Gemma Guillazo-Blanch, \\ Meritxell Torras-Garcia, Núria Boix-Trelis, and Margarita Martí-Nicolovius \\ Departament de Psicobiologia i Metodologia de les Ciències de la Salut, Institut de Neurociències, Universitat Autònoma \\ de Barcelona, Barcelona 08193, Spain
}

\begin{abstract}
The present experiments determined the consequences of blocking muscarinic cholinergic receptors of the prelimbic (PL) cortex in the acquisition and retention of an odor-reward associative task. Rats underwent a training test (five trials) and a 24-h retention test (two retention trials and two relearning trials). In the first experiment, rats were bilaterally infused with scopolamine $(20$ or $5 \mu \mathrm{g} / \mathrm{site})$ prior to training. Although scopolamine rats showed acquisition equivalent to PBS-injected controls, they exhibited weakened performance in the 24-h retention test measured by number of errors. In the second experiment, rats were injected with scopolamine $(20 \mu \mathrm{g} / \mathrm{site})$ immediately or $1 \mathrm{~h}$ after training and tested $24 \mathrm{~h}$ later. Scopolamine rats injected immediately showed severe amnesia detected in two performance measures (errors and latencies), demonstrating deficits in retention and relearning, whereas those injected $1 \mathrm{~h}$ later showed good 24-h test performance, similar to controls. These results suggest that muscarinic transmission in the PL cortex is essential for early memory formation, but not for acquisition, of a rapidly learned odor discrimination task. Findings corroborate the role of acetylcholine in consolidation processes and the participation of muscarinic receptors in olfactory associative tasks.
\end{abstract}

It is well known that acetylcholine (ACh), particularly through muscarinic receptors, is one of the most important modulators of cognitive processes (for review, see Power et al. 2003). It has been proposed that cholinergic activity participates in specific stages of memory formation; for example, ACh plays a crucial role in taste-associative tasks in signaling the novelty of the information, and shows special involvement in the early stages of memory as opposed to later phases, such as retrieval (Miranda and Bermudez-Rattoni 1999; Miranda et al. 2003). The importance of the cholinergic system in initial memory stages has been complemented with data from microdialysis studies. Learning has been associated with the increased activity of cholinergic neurons (Orsetti et al. 1996) and significant cortical ACh augmentation (Miranda et al. 2000), which appears to act on postsynaptic muscarinic receptors (Ramirez-Lugo et al. 2003). Although muscarinic activation is involved in cognitive functions such as acquisition or attention, there is also broad evidence that memory is influenced by cholinergic treatments administered after training, showing that muscarinic receptors are important in the modulation of memory consolidation (Power et al. 2003). Furthermore, increases in cortical ACh levels are observed following learning (see Power et al. 2003), which also supports the notion that ACh release contributes to memory consolidation (Gold 2003).

A region strongly modulated by cholinergic inputs affecting cognitive function is the prelimbic (PL) cortex, a component of the rat's medial prefrontal region (for review, see Boix-Trelis et al. 2007). This cortical region is involved in complex executive functions, such as attentional selectivity to relevant stimulus features during learning, working memory, and behavioral flexibility (Ragozzino et al. 1999, 2003; Dias and Aggleton 2000; Dalley et

'Corresponding author.

E-mail Anna.Vale@uab.cat; fax 34-93-5812001.

Article is online at http://www.learnmem.org/cgi/doi/10.1101//m.597507. al. 2004). Specifically related to muscarinic transmission, most experiments demonstrated participation of cholinergic muscarinic PL receptors in attention and working memory (Ragozzino 2000). However, a number of experiments from Sara's laboratory have shown that the PL, along with other brain regions, is critical for memory consolidation of a simple odor-reward association. This odor discrimination task (ODT) requires the discrimination of three odors, one of which is associated with an edible reward (Sara et al. 1999). The odor-reward association is based on the rat's spontaneous exploration behavior and is rapidly acquired in a single training session consisting of a few trials, which allows a consistent memory for at least $1 \mathrm{wk}$. It appears, therefore, wellsuited for the study of time-dependent memory formation and even initial consolidation processes (see Tronel and Sara 2002, 2003). As for PL involvement in ODT, an immunocytochemical study marking c-Fos expression demonstrated learning-specific c-Fos increases in the PL $90 \mathrm{~min}$ after ODT acquisition (Tronel and Sara 2002). Additionally, electrophysiological recordings suggested post-trial activation of PL neurons during ODT training (Kublik and Sara 2002). It should be emphasized that pharmacological approaches have shown different receptors in the PL region to have different temporal involvement in ODT memory consolidation. Thus, injections of a NMDA receptor antagonist in the PL immediately after training induced a robust and enduring amnesia (Tronel and Sara 2003), whereas injections of a noradrenergic antagonist impaired a later stage of memory consolidation (Tronel et al. 2004). Nevertheless, the contribution to ODT of other neuromodulatory systems projecting to the PL, such as the cholinergic, has not been specified.

This is an important issue, as a better understanding of the neurochemistry of medial prefrontal cortical function is necessary (Robbins 2005). In this respect, a recent study showed that $20 \mu \mathrm{g}$ of the muscarinic blocker scopolamine administered before training in the PL disrupted learning of an olfactory relational task, the social transmission of food preference (Boix-Trelis et al. 
2007). The present research further examined the involvement of the PL muscarinic receptors in the acquisition and memory formation of ODT, which may be considered another naturalistic associative olfactory task. Experiment 1 evaluated the effects of bilateral doses of scopolamine ( $20 \mu \mathrm{g}$ or $5 \mu \mathrm{g} / \mathrm{site})$ in the $\mathrm{PL}$, prior to training, on ODT acquisition and 24-h tests to determine whether muscarinic receptors are involved in ODT learning. Experiment 2 explored the consequences of injecting $20 \mu \mathrm{g}$ of scopolamine in the PL after training to assess its effects on ODT memory consolidation. Two post-training time points were considered, both immediately or $1 \mathrm{~h}$ after acquisition. These two respective intervals were chosen to determine the temporal participation of muscarinic receptors in ODT consolidation, as ACh is reported to be involved in early memory formation.

\section{Results}

\section{Experiment 1}

\section{Histology}

At the end of the experiment, the rats were subjected to histological verification of cannula placements. The thionin injection analyses indicated that the fluid spread ventral and lateral to the injection site and that it was concentrated in the intended area. For the final sample we only considered rats with their cannula tips in the PL within the area delimited by the anterior cingulate and infralimbic cortices, and in which no tissue damage due to the rate or volume of the infusions was detected (Fig. 1A). Specifically, the cannulae were located along different brain coordinates from 3.70 to $2.70 \mathrm{~mm}$ anterior to bregma (Fig. 1B) according to the stereotaxic atlas (Paxinos and Watson 1997). Twenty-seven rats were excluded from behavioral data analyses since their cannulae were unintentionally implanted outside of the PL (infralimbic, forceps minor corpus callosum, or III ventricle; $n=14$ ) or due to technical problems during the scopolamine infusion $(n=13)$. Thus, the final sample was made up of 40 subjects distributed into the following groups: SCOP20 $(n=12)$, SCOP5 $(n=15)$, and VEH $(n=13)$.

\section{Behavioral testing}

Rats were injected with scopolamine or vehicle and then trained in the ODT in five trials (grouped in two blocks: Acq123 and Acq45). The test phase, $24 \mathrm{~h}$ after acquisition, was not preceded by scopolamine injections and consisted of four trials (two blocks of two trials each: retention and relearning), the first of which was not rewarded. The analysis of latencies to find the correct sponge and make the nose-poke response for the four blocks did not show statistically significant differences between groups and the interaction Group $\times$ Block was not significant either. Nevertheless, the Block factor was statistically significant $\left(F_{(3,111)}=33.19 ; P<0.0001\right)$ (Fig. $\left.2 \mathrm{~A}\right)$. In contrast, the analysis
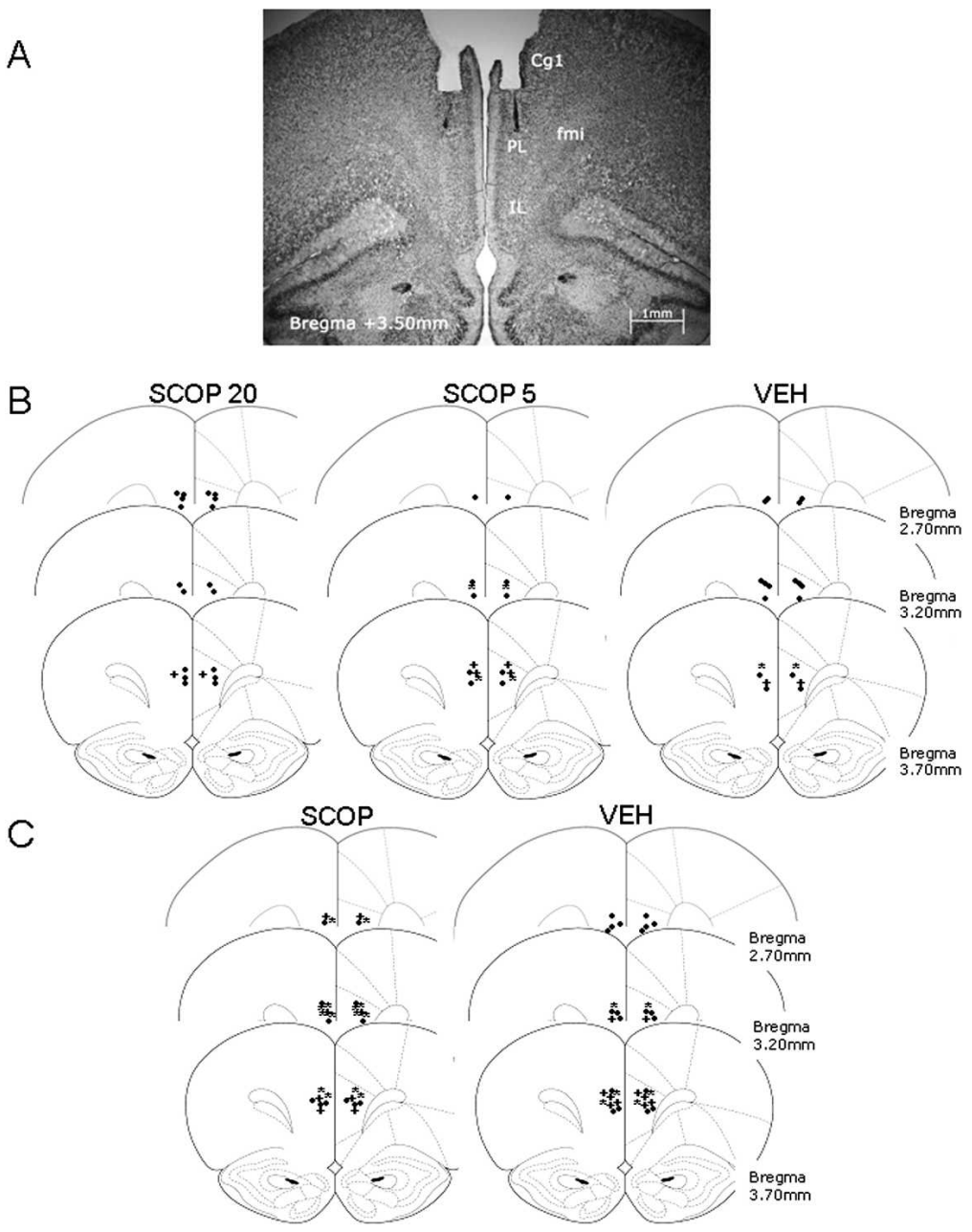

Figure 1. (A) Photomicrograph of Cresyl violet staining at the level of the PL area (AP, $3.50 \mathrm{~mm}$ Microinjector tip placements for different groups throughout the rostral-caudal extent of the $P L$ (from 3.70 to $2.70 \mathrm{~mm}$ anterior to bregma) in experiments $1(B)$ and 2 (C). Reprinted with permission from Elsevier (C) 1997, Paxinos and Watson (1997). (๑) $n=1,\left(^{*}\right) n=2,(+) n=3$. (Cg1) Cingulated cortex area 1; (fmi) forceps minor of the corpus callosum; (IL) infralimbic cortex; (PL) prelimbic cortex.

of the number of errors demonstrated that the Group and Group $\times$ Block factors tended to statistical significance $\left(F_{(2,37)}=2.73 ; P=0.079\right.$ and $F_{(6,111)}=1.91 ; P=0.086$, respectively). Also, the Block factor was highly significant $\left(F_{(3,111)}=12.88 ; P<0.0001\right)$ (Fig. 2B).

\section{Acquisition}

Rapid learning was demonstrated by the progressive decrease, in the course of acquisition trials, in latency to nose-poking the correct sponge $\left(F_{(1,37)}=63.92 ; P<0.0001\right)$ and in the number of errors $\left(F_{(1,37)}=17.76 ; P<0.0001\right)$ (Fig. 2$)$. Although muscarinic blockade was carried out before ODT training, no statistically significant between-group differences were detected in any acquisition block in terms of latencies or errors.

\section{Retention and relearning}

The mean latencies and errors for each group in the two retention and the two relearning trials of the 24 -h test can be seen in Figure 2. As for latency, no significant differences were detected in any block of the 24-h test. However, contrast analyses indicated that SCOP rats made more errors than VEH rats in retention 
A

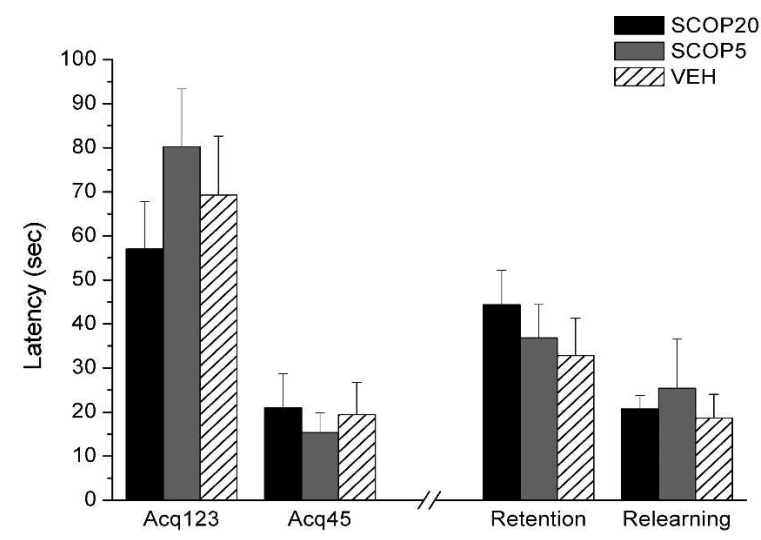

B

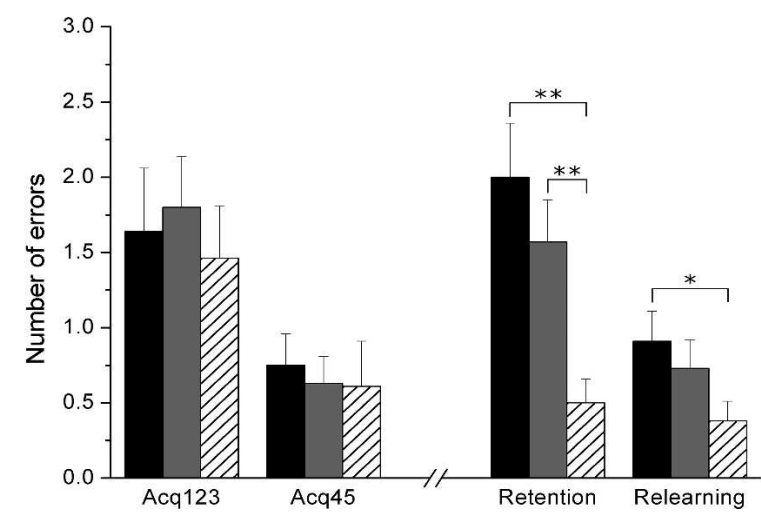

Figure 2. Pre-acquisition scopolamine injections in the prelimbic cortex. $(A)$ Latency to make the correct response ( \pm SEM) over the three first acquisition trials (Acq123), the two last acquisition trials (Acq45), the two first 24-h test trials (Retention), and the two last 24-h test trials (Relearning). All groups show similar performance both in acquisition and 24-h test. $(B)$ Number of errors before making the correct response $( \pm$ SEM) over the three first acquisition trials (Acq123), the two last acquisition trials (Acq45), the two first 24-h test trials (Retention) and the two last 24-h test trials (Relearning). All groups show similar performance in the acquisition, but both SCOP groups commit more errors than the control group in the retention phase, and the SCOP20 group in the relearning the phase. $\left(^{* *}\right) P \leq 0.001,\left(^{*}\right) P<0.05$.

$\left(\right.$ SCOP20: $F_{(1,37)}=14.07 ; P=0.001, \operatorname{SCOP} 5: F_{(1,37)}=7.94$; $P=0.008)$, and relearning (SCOP20: $\left.F_{(1,37)}=4.24 ; P=0.047\right)$. The analysis of the two types of errors (nose-poke into nonrewarded sponges-commission and failure to nose-poke after sniffing the rewarded sponge-omission) showed in retention that SCOP20 rats made significantly more commissions and omissions than VEH rats $\left(F_{(1,37)}=8.49 ; P=0.006, F_{(1,37)}=5.80\right.$; $P=0.021)$, and that SCOP5 rats made more omissions than VEH rats $\left(F_{(1,37)}=5.05 ; P=0.031\right)$.

To control for localization of the scopolamine effect on the ODT 24-h test, performances of SCOP rats discarded from the main analyses (see Histology section) and VEH rats were compared. Such an analysis demonstrated no significant differences between these groups in retention or relearning.

Performance did not seem to be related to deficits in olfactory sensitivity, since no statistically significant between-group differences were observed when the latency to find a buried cookie was analyzed (Fig. 3).

\section{Experiment 2}

In order to analyze the role of PL muscarinic transmission in ODT consolidation, rats in Experiment 2 were injected with scopol- amine in the PL immediately or $1 \mathrm{~h}$ after ODT training and tested $24 \mathrm{~h}$ later. A 20- $\mu \mathrm{g}$ dose was used, as it was the most effective to increase the number of errors in Experiment 1.

\section{Histology}

The cannula implantations were similar to those described for Experiment 1 (see Fig. 1C). Twelve rats were excluded from behavioral data analyses according to the same criteria as in Experiment 1 . Thus, the final sample was made up of 63 subjects distributed into the following groups according to drug (scopolamine or vehicle) and microinfusion delay (immediate or $1 \mathrm{~h}$ ): SCOP-Immed $(n=18)$, VEH-Immed $(n=15)$, SCOP-1h $(n=14)$, and VEH-1h $(n=16)$.

\section{Behavioral testing}

Rats were trained in the ODT in five trials (Acq123 and Acq45), and were then injected with scopolamine. The 24-h test consisted of four trials (retention, two trials, and relearning, two trials) as in Experiment 1. In both performance measures, latency and number of errors, there were statistically significant effects of Group $\left(F_{(3,59)}=6.31 ; P=0.001, F_{(3,59)}=4.10 ; P=0.01\right)$, Block $\left(F_{(3,177)}=14.03 ; P<0.0001, F_{(3,177)}=10.23 ; P<0.0001\right)$, and Group $\times$ Block $\left(F_{(9,177)}=4.63 ; P<0.0001, F_{(9,177)}=4.54\right.$; $P<0.0001$ ) (Fig. 4).

\section{Acquisition}

Rapid learning was observed by the progressive decrease in latency to make the nose-poking response $\left(F_{(1,59)}=69.82\right.$; $P<0.0001)$ and the progressive decrease in the number of errors $\left(F_{(3,177)}=24.28 ; P<0.0001\right)$. No between-group differences were found in any acquisition measure (Fig. 4).

\section{Retention and relearning}

As seen in Figure 4, scopolamine administered immediately after acquisition impaired performance in the 24-h test. Regarding latency to make the correct response (Fig. 4A), SCOP-Immed rats showed significantly more latency in nose-poking the rewarded sponge than VEH-Immed rats both in retention $\left(F_{(1,59)}=10.71\right.$; $P=0.002)$ and relearning $\left(F_{(1,59)}=5.79 ; P=0.019\right)$. Differences were also detected between SCOP-Immed and SCOP-1h both in retention $\left(F_{(1,59)}=13.70 ; P<0.0001\right)$ and relearning $\left(F_{(1,59)}=5.43 ; P=0.023\right)$. However, there were no significant differences between SCOP-1h and VEH-1h in any block.

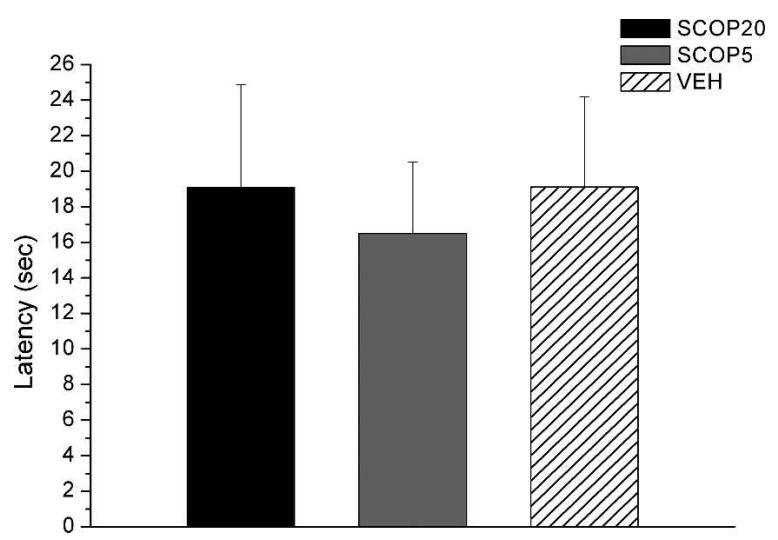

Figure 3. Latency to find the buried cookie $( \pm S E M)$ in the olfactory perception test. SCOP groups show similar latencies to the control group. 
A

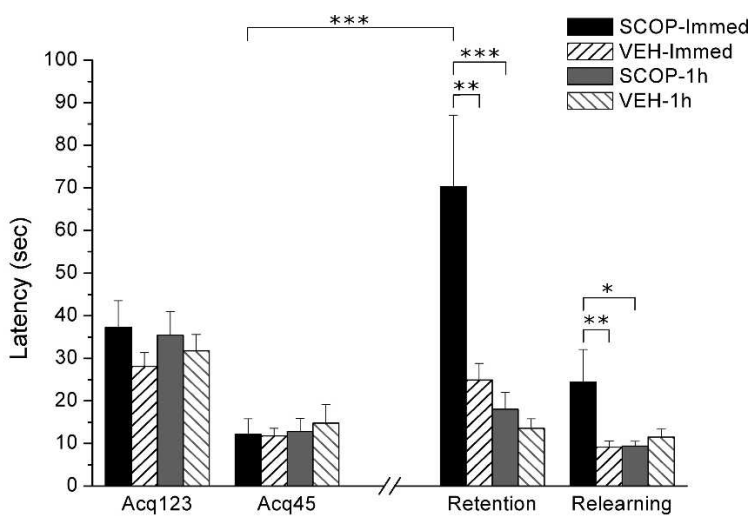

B

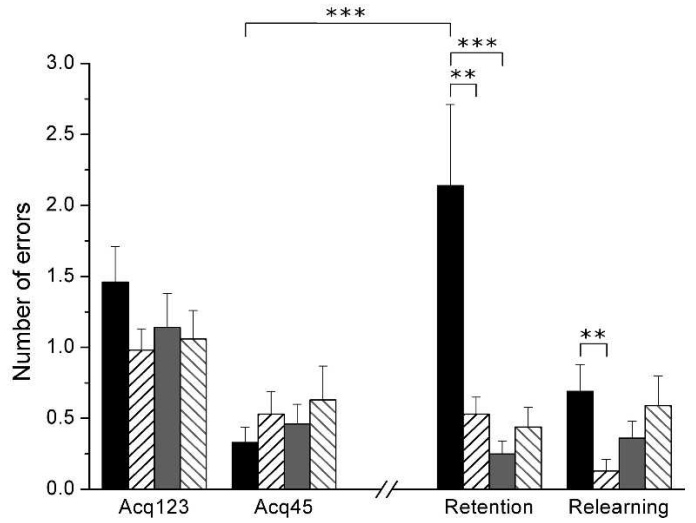

Figure 4. Post-acquisition scopolamine injections (immediately or $1 \mathrm{~h}$ after) in the prelimbic cortex. $(A)$ Latency to make the correct response ( \pm SEM) over the three first acquisition trials (Acq123), the two last acquisition trials (Acq45), the two first 24-h test trials (Retention), and the two last 24-h test trials (Relearning). The SCOP-Immed group demonstrates more latency to nose-poking than VEH-Immed and SCOP-1h both in retention and relearning. (B) Number of errors before making the correct response ( \pm SEM) over the three first acquisition trials (Acq123), the two last acquisition trials (Acq45), the two first 24-h test trials (Retention), and the two last 24-h test trials (Relearning). The SCOP-Immed group commit more errors than the VEH-Immed group both in retention and relearning and also the SCOP-1h group in retention. The SCOPImmed group significantly impaired performance from Acq45 to Retention, indicated by higher latencies and more errors. $\left.{ }^{* * *}\right) P<0.0001 ;\left({ }^{* *}\right)$ $P \leq 0.01 ;\left(^{*}\right) P<0.05$.

A similar pattern of results is shown when errors are analyzed (Fig. 4B). In retention, there were differences between SCOP-Immed group and both VEH-Immed and SCOP-1h groups $\left(F_{(1,59)}=11.58 ; P=0.001\right.$ and $F_{(1,59)}=15.43 ; P<0.0001$, respectively) and also in relearning between SCOP-Immed and VEHImmed $\left(F_{(1,59)}=6.05 ; P=0.017\right)$. In retention, SCOP-Immed rats made significantly more commissions and omissions than both VEH-Immed $\left(F_{(1,59)}=9.17 ; P=0.004, F_{(1,59)}=9.68 ; P=0.003\right)$ and SCOP-1h rats $\left(F_{(1,59)}=11.12 ; P=0.001, F_{(1,59)}=13.92\right.$; $P<0.0001)$. In relearning, SCOP-Immed rats also made more commissions and omissions than VEH-Immed rats $\left(F_{(1,59)}=4.47\right.$; $\left.P=0.039, F_{(1,59)}=7.39 ; P=0.009\right)$.

Moreover, the SCOP-Immed group showed significantly more latency to nose-poking and made more total errors in the retention trials compared with the last acquisition trials (Acq45) (Latencies: $F_{(1,59)}=39.56 ; P<0.0001$ and Errors: $F_{(1,59)}=31.95$; $P<0.0001)$, in contrast with the remaining groups, which did not show such a decrease in performance.

The low number of SCOP-Immed rats eliminated due to histological criteria $(n=2)$ did not allow for comparisons of their performance with that of VEH rats in order to control for the specific effect of scopolamine in the PL.

\section{Discussion}

Present experiments report, for the first time, that muscarinic receptors are involved in an early consolidation phase of memory for a simple odor-reward association, but do not appear indispensable to the acquisition of the task. Experiment 1 indicated that rats injected with scopolamine (5 $\mu \mathrm{g}$ or $20 \mu \mathrm{g})$ in the PL before training normally acquired the task, but made more errors than the control rats in an ODT test conducted $24 \mathrm{~h}$ later. Both SCOP groups showed poorer retention than the VEH group, and SCOP20 had an inferior relearning ability regarding the total number of errors. The deficit in retention shown by the rats infused with the higher dose seems to be unspecific regarding the type of errors (commissions and omissions), while the rats receiving the lower dose of scopolamine only showed more omissions. Results from Experiment 1 may indicate a role of PL muscarinic receptors in ODT memory formation, which was confirmed in Experiment 2. Experiment 2 showed that 20 - $\mu$ g scopolamine injections in the PL cortex immediately after ODT learning produced amnesia in retention and difficulties in relearning when the rats were tested $24 \mathrm{~h}$ later. Latencies and the two types of errors were affected, indicating that scopolamine infused without delay after training showed a remarkably disruptive effect on the whole 24-h session. Moreover, the performances in the first retention trials for the SCOP-Immed group were significantly poorer than in the final acquisition trials. The scopolamine effects are time dependent, as this treatment delayed for $1 \mathrm{~h}$ after training did not affect ODT retention or relearning. All of the above would suggest that muscarinic blockade in the PL may entirely affect the initial stages of ODT memory consolidation.

Although the present experiments were not specifically aimed at evaluating the persistence of scopolamine-induced amnesia, the deficits do not seem to be transient. Rats injected immediately after training showed a strikingly poorer performance throughout the four trials in the 24-h test compared with controls and rats injected $1 \mathrm{~h}$ after training, indicating that the deficits were robust and lasted for at least $24 \mathrm{~h}$. However, considering that SCOP20 (Experiment 1) and SCOP-Immed (Experiment 2) started from a large number of errors in retention, and that no effects were found on acquisition (Experiment 1), it may be considered that the differences between SCOP and VEH rats in relearning would possibly disappear with further relearning trials. When comparing both experiments, it can be noted that the vehicle group from the first experiment showed more latency to make the correct response in acquisition than vehicle rats from the second experiment. This may be due to the fact that rats from Experiment 1 were injected just before acquisition. Since the injection involves restraint, such a stressful procedure may have produced a slowing-down effect on performance. Nevertheless, by Acq45, vehicle rats from both experiments showed similar latencies, indicating that the possible effect of previous manipulation would seem momentary. Also, the differences in the total number of errors are much smaller, as all of the control animals committed few errors.

Current findings are broadly consistent with studies that have implicated ACh in olfactory memories. Thus, disruption of normal cholinergic function has frequently been implicated in interference of many forms of odor memory, such as simple habituation (Hunter and Murray 1989), perceptual learning (Fletcher and Wilson 2002; Wilson et al. 2004), and odor rule or set learning (Saar et al. 2001). Of significant relevance to the current study is the fact that ACh also modulates olfactory associative memory, with both lesions of the cortical cholinergic pro- 
jection neurons (Roman et al. 1993; Linster et al. 2001; ValeMartínez et al. 2002) and pharmacological receptor blockade (Ravel et al. 1994; De Rosa and Hasselmo 2000) capable of impairing several tasks. The present results, together with those reported earlier, suggest that disturbance of cholinergic function-and, in particular, muscarinic transmission-may have a significant impact on different olfactory memory tasks.

Some considerations should be made in order to clarify our results, especially those from Experiment 1 . Firstly, it is important to determine whether the impairments in errors reported in ODT retention and relearning reflect decreases in olfactory sensitivity. In our research, odor-detection performance was not affected by infusions of scopolamine, as shown in the olfactory perception test. Such data agree with a recent study reporting that systemic administration of different doses of scopolamine, which are known to influence odor memory and learning, did not cause alterations in the odor-detection performance of rats (Doty et al. 2003). Secondly, the duration of the scopolamine action in the brain is an aspect to consider in the explanation of our findings. In Experiment 1, SCOP animals were trained under the influence of scopolamine and tested $24 \mathrm{~h}$ after training in an "off-drug" state. As effective doses of anticholinergic drugs have been shown to produce state-dependent learning, we cannot absolutely rule out the possibility of state-dependent learning as an explanation for the impairment in an ODT 24-h test. It has been reported, however, that scopolamine induced state dependency only after over-reinforcement, when high systemic doses were used (Quirarte et al. 1994). Considering that our training was accomplished in only five trials, and that Experiment 2 demonstrated an undoubted effect on ODT consolidation, a statedependent learning interpretation would not appear to be the timeliest. Thirdly, specificity of the scopolamine effect in the PL cortex and not in the surrounding areas may be inferred from the fact that rats injected in the vicinity of the PL and controls showed similar performances in the 24 -h test. Taken together, the most plausible explanation would be that scopolamine in the PL may have influenced brain processes underlying the early storage of new odorous information, confirmed by an amnesic effect of the 24-h test in the second experiment.

Experiment 1 indicated that muscarinic cholinergic transmission in the PL cortex is not essential for discrimination learning based on odorous stimuli. This is in accordance with studies showing that the blockade of muscarinic receptors in the PL does not impair visuospatial discriminations (Ragozzino 2000). Indeed, the studies analyzing the effects of scopolamine infusions specifically in the rat medial prefrontal cortex mainly implicate muscarinic transmission in working memory or short-term memory (Broersen et al. 1995; Granon et al. 1995; Ragozzino and Kesner 1998; Chudasama et al. 2004) and attention (Robbins et al. 1998). As scopolamine in Experiment 1 was administered before behavioral testing, it may have acted by influencing such processes. However, scopolamine administered before training did not prevent a rapid learning of the task (Experiment 1). Moreover, in Experiment 2, muscarinic activation remained influential in memory after the animals were no longer attending to a new training experience. In view of both this and the fact that ODT does not specifically tax working memory or attention, an interpretation of results from Experiment 1 purely derived from impairments in such cognitive functions would not be the most appropriate. In fact, the present experiments suggest that muscarinic action in the PL is not critical for olfactory discrimination (Experiment 1), and it is not limited to working memory or attention, but is involved in processing information for early memory consolidation of a simple odor-reward task (Experiment 2).

Previous evidence indicated that cortical muscarinic activa- tion is a critical component in the modulation of memory consolidation for several kinds of tasks, apart from other possible influences on acquisition or attention. In this respect, it has been demonstrated that antimuscarinic treatments administered posttraining, in the cingulated or insular cortices, disrupted consolidation of new memories in paradigms such as inhibitory avoidance (Riekkinen et al. 1995; Miranda and Bermudez-Rattoni 2007) or familiarization with a novel gustatory stimulus (Gutierrez et al. 2003). It has also been shown that cholinergic projections from the nucleus basalis to the cortex are required for posttraining amygdalar modulation of memory storage processes in an inhibitory avoidance task (Power et al. 2002). Consistent with such findings, ACh levels in the cortex have been reported to be enhanced after a spatial discrimination (Toumane et al. 1988) and an operant conditioning (Orsetti et al. 1996). Such increases were also observed in the hippocampus, but persisted longer in the cortex (prefrontal and parietal). Additionally, muscarinic receptors contribute to memory formation, principally through postsynaptic receptors. Thus, taste-aversion initial memory formation was abolished by injections of pirenzepine (a postsynaptic M1 and M3 receptors antagonist) but not AFDX 116 (a pre-synaptic M2 receptor antagonist) (Ramirez-Lugo et al. 2003). Present results also agree with findings demonstrating that M1 receptor knockout mice had pronounced consolidation deficits in the absence of acquisition deficits (Anagnostaras et al. 2003). To address the issue of how the specific cellular effects of ACh within cortical structures could underlie the role of ACh in the encoding of new memories, it has been proposed that ACh increases the strength of afferent input relative to feedback (via muscarinic presynaptic inhibition of excitatory feedback synapses). ACh can also activate intrinsic mechanisms for persistent spiking of individual cortical neurons, which could provide a mechanism for maintenance of information both for short-term memory and encoding of information into long-term memory (Hasselmo 2006).

The effective site of action of the scopolamine injection in the PL confirms the importance of this cortical area in ODT. The PL cortex has reciprocal connections to olfactory bulbs (Neafsey et al. 1986) and piriform cortex (Datiche and Cattarelli 1996), and it is activated after learning the ODT task (Tronel and Sara 2002). Other studies indicate that NMDA receptors in the PL region are also important in the early stages of ODT memory consolidation, reporting a pattern of results much similar to the present findings. The NMDA antagonist APV injected in the PL immediately after training (as opposed to $2 \mathrm{~h}$ ) disrupted the retention and slowed down the relearning of the task, both tested $48 \mathrm{~h}$ after training (Tronel and Sara 2003). There are also consistent observations as to the involvement of the noradrenergic system in ODT memory consolidation. The blockade of $\beta$-adrenergic receptors in the PL $2 \mathrm{~h}$ after training (but not $5 \mathrm{~min}$ ) induced a profound amnesia assessed $48 \mathrm{~h}$ later (Tronel et al. 2004). Together, previous data and present results suggest that different neurochemical mechanisms may subserve different ODT memory phases. Accordingly, glutamatergic and cholinergic systems may contribute to the initial stages of memory formation, whereas noradrenaline may have a delayed role in a late phase of ODT long-term memory consolidation.

One of the most remarkable interactions between neurotransmitter systems is the inter-related activity between ACh and glutamate to regulate neural plasticity. Plasticity in the visual, somatosensory, and auditory sensory cortices requires engagement of muscarinic receptors (Delacour et al. 1990; Kirkwood et al. 1999; Miasnikov et al. 2001). Muscarinic receptors may mediate cortical plasticity via glutamate receptors, activating second messenger systems (Cox et al. 1994), G protein-mediated events in the post-synaptic cell related to memory formation (Aramakis 
et al. 1997). Therefore, it is tempting to suggest that these two neurotransmitter systems may act cooperatively in the PL cortex to regulate early ODT memory formation, initiating intracellular events related to plastic changes. A comparable interpretation has been proposed for the gustatory memory consolidation in the insular cortex (Miranda et al. 2003). There are also a number of studies stressing the synergistic interaction between cholinergic muscarinic and glutamatergic NMDA receptors in the modulation of some forms of learning and memory. Thus, blockade of cholinergic and glutamatergic systems induced drastic memory impairments, stronger than blockade of either system alone (Ohno and Watanabe 1996; Li et al. 1997; Hlinak and Krejci 1998; Monteiro Moreira et al. 2005). In this respect, it has been suggested that one of ACh's actions is to modulate hippocampal and cortical neurons, thereby reducing interference so that new associations can be formed from afferent inputs utilizing glutamate (Aigner 1995).

The involvement in odor-reward associative learning of cortical regions other than PL, hippocampal, and other subcortical structures has also been studied. A study using c-Fos immunoreaction showed significant post-training activation of orbital cortex and basolateral amygdala, but not central amygdala (Tronel and Sara 2002). These regions, along with prelimbic, piriform, and infralimbic cortices, are thought to act in concert to link sensory (olfactory) and emotional (reward) information (Ongur and Price 2000). However, the two latter cortical regions did not show increases in post-training c-Fos expression (Tronel and Sara 2002). As for the hippocampal involvement in ODT, injections of an NMDA antagonist into the dorsal hippocampus had no effect on retention, consistently with the observation that hippocampal regions (CA1, CA3, DG) showed no learning-related c-Fos activity (Tronel and Sara 2002, 2003). In accordance with such evidence, we suggest that muscarinic blockade in orbitofrontal cortex or basolateral amygdala might disrupt ODT memory formation. Moreover, it would be interesting to study whether scopolamine applied in the lateral habenula might affect retention or retrieval, as it showed strong activation after ODT retrieval (Tronel and Sara 2002).

In conclusion, the current research indicates that muscarinic receptors may play an important role in the regulation of early stages of ODT memory formation in the PL cortex. Such receptors seem to be essential for good consolidation during a precise time window; possibly from the initial phases of learning to several minutes after learning, but are not required $1 \mathrm{~h}$ after training to show a normal ODT performance $24 \mathrm{~h}$ later. Further knowledge is needed to ascertain interactions in the PL cortex between the muscarinic and other neurotransmitter receptors, such as NMDA, and the molecular events through which ACh modulates this odor-reward association. More data are also needed as to the effects of muscarinic blockade of other brain regions to determine the cholinergic circuit underlying the formation of ODT memory.

\section{Materials and Methods}

\section{Experiment 1}

\section{Subjects}

Sixty-seven male Wistar rats obtained from our laboratory breeding stock with a mean age of $94.39 \mathrm{~d}(\mathrm{SD}=6.14)$ and a mean weight of $436.29 \mathrm{~g}(\mathrm{SD}=39.33)$ at the beginning of the experiment were used. All rats were housed singly in $22 \times 22 \times 14-\mathrm{cm}$ plastic-bottomed cages with sawdust bedding, kept under conditions of controled temperature $\left(20^{\circ} \mathrm{C}-23^{\circ} \mathrm{C}\right)$ and humidity $(40 \%-$ $70 \%$ ), and maintained on a 12-h light-dark cycle (lights on at 8:00 a.m.). Experiments were performed during the light phase of the cycle. Rat-chow pellets (Panlab S.L, A04) and water were pro- vided ad libitum except during habituation, training, and testing sessions, when rats were submitted to a food restriction schedule $(12 \mathrm{~g} /$ day, to maintain body weight at $85 \%$ of freely feeding weight). The animals were handled daily for $\sim 10 \mathrm{~min}$ and restrained for $2 \mathrm{~min}$ to habituate them to the injection procedure. All of the procedures were carried out in compliance with the European Community Council Directive for care and use of laboratory animals (86/609/ECC) and with the Generalitat de Catalunya authorization (DOGC 2073 10/7/1995, DARP protocol number 3211).

\section{Surgery}

Animals were anesthetized and underwent stereotaxic implantation of chronic double-guide cannulae following procedures explained in detail elsewhere (Boix-Trelis et al. 2007). Each guide cannula comprised two 26-gauge metal tubes that were $1.2 \mathrm{~mm}$ apart, projecting $2.9 \mathrm{~mm}$ from the pedestal (Plastics One, Bilaney Consultants GMBH). The stereotaxic coordinates for the PL were: $\mathrm{AP},+3.5 \mathrm{~mm}$ from bregma; $\mathrm{ML}, \pm 0.6 \mathrm{~mm}$ from midline; and $\mathrm{DV}$, $-2.9 \mathrm{~mm}$ from cranium surface (Paxinos and Watson 1997). Sterile dummy stylets (Plastics One) were placed into the cannulae to prevent occlusion. Each rat received buprenorphine (Buprex, Schering-Plough S.A.) to reduce post-surgical pain (s.c. $0.5 \mathrm{mg} / \mathrm{kg}$ ). After surgery, rats were returned to home cages for $7 \mathrm{~d}$ before behavioral training ( $3 \mathrm{~d}$ for recovery, $3 \mathrm{~d}$ for food restriction, and $1 \mathrm{~d}$ for habituation). During the 7-d recovery period, rats were handled and weighed daily and the dummy stylets were changed every other day.

\section{Microinfusion procedure}

Immediately before ODT training, the rats were gently restrained while the dummy stylets were removed and replaced with a 33gauge stainless-steel double injector (Plastics One) extending $1 \mathrm{~mm}$ below the cannula tips. The double injectors were connected by polyethylene tubing (Plastics One) to two $5-\mu \mathrm{L}$ syringes (Hamilton, Microliter Syringes) mounted in an infusion pump (11 Plus Syringe Pump, Harvard Apparatus Inc.). Scopolamine (Scopolamine Hydrobromide USP, Sigma-Aldrich) was dissolved in PBS (0.1 M at pH 7.4) in two different doses: $20 \mu \mathrm{g} /$ hemisphere (SCOP20 group) and $5 \mu \mathrm{g} / \mathrm{hemisphere} \mathrm{(SCOP5}$ group). The rats in the control VEH group received vehicle (PBS) injections. The solutions were infused bilaterally in a volume of $0.5 \mu \mathrm{L} /$ hemisphere for $2 \mathrm{~min}$. The injectors were left in place for $1 \mathrm{~min}$ after the infusion was complete to allow for diffusion.

\section{Apparatus}

The training apparatus and behavioral procedures are explained elsewhere (Quiroz-Padilla et al. 2007). The training box contained three sponges with a $3-\mathrm{cm}$ diameter hole cut into the center, placed in glass slide holders of the same size. The food reinforcement used was a crispy chocolate rice breakfast cereal (Kellogg's) that was placed at the bottom of the opening in the sponge. Each sponge was impregnated with an odor by placing the essence on each of its corners. Odors used were vanilla $(0.2 \mathrm{~mL})$, orange $(0.6 \mathrm{~mL})$, and anise $(0.3 \mathrm{~mL})$ (Vahiné). All behavioral sessions were recorded by a video camera (Panasonic NV-RX22EG) connected to a monitor.

\section{Behavioral procedures}

Habituation sessions

Rats were submitted to three habituation sessions before surgery to familiarize them with the reinforcement and the training box, and they were food-deprived for $5 \mathrm{~d}$ before the first habituation session. In the habituation sessions, the rats were given free access to the reinforcement in a plastic bottomed cage $(50 \times 22 \times 14 \mathrm{~cm})$ until they consumed 10 pieces of cereal. The rats were then placed in the training box and allowed to explore it for $45 \mathrm{~min}$ on the first day, $30 \mathrm{~min}$ the second day, and $15 \mathrm{~min}$ the third day. Six days after surgery, rats were food-deprived again and submitted to an identical 15-min habituation period. The same day, rats were also adapted to a mock infusion protocol 
(no solutions injected) in order to minimize any stress associated with the procedure.

\section{Acquisition session}

One day after the post-surgery habituation, rats received a bilateral intracerebral infusion of $20 \mu \mathrm{g}$ of scopolamine, $5 \mu \mathrm{g}$ of scopolamine, or PBS in the PL immediately before ODT training. Training was carried out in a single five-trial session, according to procedures previously described (Quiroz-Padilla et al. 2007). The trials were grouped into two blocks: Acq123 (when learning was in progress) and Acq45 (when learning was more stable and reached an asymptotic level) (Sara et al. 1999). The reinforcement was chocolate rice cereal placed at the bottom of the opening in the target sponge. The reinforcement was associated with the same odor across trials, and the target odor was randomly assigned to each rat in a counterbalanced way for all groups. The sponges with the nonreinforced odors did not contain food. Sponges were placed in any three of the four corners of the box, and the position of each odor within the box was changed for each trial according to a previously determined protocol.

The rats were placed in the training box, in the corner without a sponge, with their heads facing the wall. There was a 6-min limit for the rat to find and consume the reinforcement and the intertrial interval was $1 \mathrm{~min}$. Latency before a correct response (nose-poking into the target sponge) and number of errors were scored. Two different errors were combined: errors of commission (nose-poking into a non-target sponge) and omissions (sniffing the target sponge not followed by nose-poking) (Tronel and Sara 2003).

\section{Twenty-four hour test session}

Twenty-four hours after training, rats were tested for retention and relearning ability using the same procedure as during training, except for the fact that the first test trial was not reinforced and there were four trials in total. The first trial served as a direct measure of memory of the previous training. Since the first trial was not reinforced, the second trial reflected resistance to extinction, which is considered an indirect measure of retention (Sara et al. 1999; Tronel and Sara 2003; Torras-Garcia et al. 2005). The last two trials were an index of the rats' relearning ability (Sara et al. 1999; Tronel and Sara 2003; Torras-Garcia et al. 2005). Latencies before correct responses and number of errors were also scored during this test.

\section{Olfactory perception test}

To rule out olfactory alterations due to the scopolamine infusions, an additional olfactory perception test was conducted at the end of the experiment (Wrenn et al. 2003; Quiroz-Padilla et al. 2006) on a sample of each group (SCOP20, $n=10$; SCOP5, $n=8$; and VEH, $n=9$ ). Twenty-four hours before the olfactory test, rats were habituated to butter-flavored cookies (Brambly Hedge). The rats were then food restricted for $24 \mathrm{~h}$ prior to the infusion and the test. Immediately before the test, rats were infused with scopolamine $(20 \mu \mathrm{g}$ or $5 \mu \mathrm{g})$ or PBS. The test was conducted in clean rat cages $(50 \times 22 \times 14 \mathrm{~cm})$ and a piece of cookie was buried in one corner of the cage. The rat was then placed in the cage, and the latency to find the buried cookie and commence eating was timed.

\section{Histology}

Upon completion of the behavioral study, SCOP20, SCOP5, and VEH rats were deeply anesthetized with an overdose of sodium pentobarbital (Dolethal, $200 \mathrm{mg} / \mathrm{kg}$; Vetoquinol S.A.). Previously, a subset of rats received a $0.5-\mu \mathrm{L}$ infusion of $5 \%$ thionin (Thionin acetate, Sigma-Aldrich) through each guide cannula. Rats were perfused transcardially with $0.9 \%$ saline followed by $10 \%$ formalin. The cannulae were carefully removed and brains postfixed in formalin for at least $24 \mathrm{~h}$ and then submerged in a 30\% sucrose solution prior to sectioning. Coronal $40-\mu \mathrm{m}$ sections were cut on a cryostat (Shandom Cryotome FSE, Thermo Electron Corporation), mounted, and stained with Cresyl violet. The sections were examined to verify cannula placement by two independent observers under a light microscope (Olympus BX 41; Olympus Op- tical CO, LTD). Microphotographs of the cannula placements were taken with a digital camera (Olympus DP70).

\section{Data analysis}

The analysis was carried out by means of a $3 \times 4$ mixed analyses of variance (ANOVA; SPSS v14), in which the between-factor was Group (SCOP20, SCOP5, and VEH) and the within-factor was Block. The Block factor consisted of four measures: Acq123 (the average scores for the first three trials of the acquisition), Acq45 (the average scores for the last two trials of the acquisition), Retention (the average scores for the first two trials of the 24-h test) and Relearning (the average scores for the last two trials of the 24-h test). The dependent variables were Latencies and Errors. Corresponding contrasts were performed when necessary.

Regarding the olfactory test, an additional ANOVA analysis was applied considering Group (SCOP20, SCOP5, and VEH) as the independent variable, and Latency in finding the buried cookie as the dependent variable.

\section{Experiment 2}

\section{Subjects}

Seventy-five male Wistar rats obtained from our laboratory breeding stock with a mean age of $97.25 \mathrm{~d}(\mathrm{SD}=3.28)$ and a mean weight of $422.85 \mathrm{~g}(\mathrm{SD}=38.50)$ at the beginning of the experiment were used. The animals were housed and maintained under the same conditions described for Experiment 1.

\section{Surgery}

The surgical protocol and stereotaxic coordinates were identical to Experiment 1.

\section{Microinfusion procedure}

The microinfusion procedures were the same as in Experiment 1, but only a $20-\mu \mathrm{g}$ dose of scopolamine was administered to rats in the scopolamine groups. The rats in the control groups received PBS injections. SCOP-Immed and VEH-Immed rats were injected immediately after the last training trial, whereas SCOP- $1 \mathrm{~h}$ and $\mathrm{VEH}-1 \mathrm{~h}$ rats received microinfusions $1 \mathrm{~h}$ after training. During the training-to-injection interval the rats were returned to their home cage.

\section{Behavioral procedures}

Habituation sessions

All behavioral procedures were similar to those described for Experiment 1 . Rats were submitted to three habituation sessions before surgery and to a fourth one before ODT training.

\section{Acquisition session}

Training was carried out in the same way as Experiment 1, except for the fact that bilateral PL infusions were administered after the last training trial, at two different delays in separate groups (immediately or $1 \mathrm{~h}$ afterward).

\section{Twenty-four hour test session}

Twenty-four hours after training, all rats were tested for retention and relearning ability as in the first experiment.

\section{Histology}

The histological procedures and the criteria applied were the same as in Experiment 1.

\section{Data analysis}

Similarly to Experiment 1 , the main analyses of the acquisition data were carried out by means of $4 \times 4$ mixed ANOVA, with the independent variable Group (SCOP-Immed, VEH-Immed, SCOP$1 \mathrm{~h}$, and VEH-1h) and the dependent variables Latencies and Errors. Regarding the Block within-factor, two blocks of trials were considered in both the acquisition (Acq123 and Acq45) and the $24 \mathrm{~h}$ test (Retention and Relearning). Corresponding betweenand within-group contrasts were also planned. 


\section{Acknowledgments}

This research was supported by funds provided by Generalitat de Catalunya (SGR2005-00551, A.C.M.: 2005FI 00655, 2006FIC 00200) and by the Ministerio de Educación y Ciencia (SEJ200502518). We thank Gerald-Patrick Fannon for revising the manuscript. Some of these data were presented at the Eight Conference on the Neurobiology of Learning and Memory (Irvine, USA; March 2006).

\section{References}

Aigner, T.G. 1995. Pharmacology of memory: Cholinergic-glutamatergic interactions. Curr. Opin. Neurobiol. 5: 155-160.

Anagnostaras, S.G., Murphy, G.G., Hamilton, S.E., Mitchell, S.L., Rahnama, N.P., Nathanson, N.M., and Silva, A.J. 2003. Selective cognitive dysfunction in acetylcholine M1 muscarinic receptor mutant mice. Nat. Neurosci. 6: 51-58.

Aramakis, V.B., Bandrowski, A.E., and Ashe, J.H. 1997. Activation of muscarinic receptors modulates NMDA receptor-mediated responses in auditory cortex. Exp. Brain Res. 113: 484-496.

Boix-Trelis, N., Vale-Martínez, A., Guillazo-Blanch, G., and Marti-Nicolovius, M. 2007. Muscarinic cholinergic receptor blockade in the rat prelimbic cortex impairs the social transmission of food preference. Neurobiol. Learn. Mem. 87: 659-668.

Broersen, L.M., Heinsbroek, R.P., de Bruin, J.P., Uylings, H.B., and Olivier, B. 1995. The role of the medial prefrontal cortex of rats in short-term memory functioning: Further support for involvement of cholinergic, rather than dopaminergic mechanisms. Brain Res. 674: $221-229$.

Chudasama, Y., Dalley, J.W., Nathwani, F., Bouger, P., and Robbins, T.W. 2004. Cholinergic modulation of visual attention and working memory: Dissociable effects of basal forebrain 192-IgG-saporin lesions and intraprefrontal infusions of scopolamine. Learn. Mem. 11: $78-86$.

Cox, C.L., Metherate, R., and Ashe, J.H. 1994. Modulation of cellular excitability in neocortex: Muscarinic receptor and second messenger-mediated actions of acetylcholine. Synapse 16: 123-136.

Dalley, J.W., Cardinal, R.N., and Robbins, T.W. 2004. Prefrontal executive and cognitive functions in rodents: Neural and neurochemical substrates. Neurosci. Biobehav. Rev. 28: 771-784.

Datiche, F. and Cattarelli, M. 1996. Reciprocal and topographic connections between the piriform and prefrontal cortices in the rat: A tracing study using the B subunit of the cholera toxin. Brain Res. Bull. 41: 391-398.

De Rosa, E. and Hasselmo, M.E. 2000. Muscarinic cholinergic neuromodulation reduces proactive interference between stored odor memories during associative learning in rats. Behav. Neurosci. 114: $32-41$.

Delacour, J., Houcine, O., and Costa, J.C. 1990. Evidence for a cholinergic mechanism of "learned" changes in the responses of barrel field neurons of the awake and undrugged rat. Neuroscience 34: $1-8$.

Dias, R. and Aggleton, J.P. 2000. Effects of selective excitotoxic prefrontal lesions on acquisition of nonmatching- and matching-to-place in the T-maze in the rat: Differential involvement of the prelimbic-infralimbic and anterior cingulate cortices in providing behavioural flexibility. Eur. J. Neurosci. 12: 4457-4466.

Doty, R.L., Bagla, R., Misra, R., Mueller, E., and Kerr, K.L. 2003. No influence of scopolamine hydrobromide on odor detection performance of rats. Chem. Senses 28: 761-765.

Fletcher, M.L. and Wilson, D.A. 2002. Experience modifies olfactory acuity: Acetylcholine-dependent learning decreases behavioral generalization between similar odorants. J. Neurosci. 22: RC201.

Gold, P.E. 2003. Acetylcholine modulation of neural systems involved in learning and memory. Neurobiol. Learn. Mem. 80: 194-210.

Granon, S., Poucet, B., Thinus-Blanc, C., Changeux, J.P., and Vidal, C. 1995. Nicotinic and muscarinic receptors in the rat prefrontal cortex: Differential roles in working memory, response selection and effortful processing. Psychopharmacology 119: 139-144.

Gutierrez, R., Tellez, L.A., and Bermudez-Rattoni, F. 2003. Blockade of cortical muscarinic but not NMDA receptors prevents a novel taste from becoming familiar. Eur. J. Neurosci. 17: 1556-1562.

Hasselmo, M.E. 2006. The role of acetylcholine in learning and memory. Curr. Opin. Neurobiol. 16: 710-715.

Hlinak, Z. and Krejci, I. 1998. Concurrent administration of subeffective doses of scopolamine and MK-801 produces a short-term amnesia for the elevated plus-maze in mice. Behav. Brain Res. 91: 83-89.

Hunter, A.J. and Murray, T.K. 1989. Cholinergic mechanisms in a simple test of olfactory learning in the rat. Psychopharmacology 99: 270-275.
Kirkwood, A., Rozas, C., Kirkwood, J., Perez, F., and Bear, M.F. 1999. Modulation of long-term synaptic depression in visual cortex by acetylcholine and norepinephrine. J. Neurosci. 19: 1599-1609.

Kublik, E. and Sara, S.J. 2002. Activity in medial frontal cortex during odour discrimination learning in the rat: Neuronal response to experimental cortex. FENS Abstract 1, A040.14.

Li, H.B., Matsumoto, K., Tohda, M., Yamamoto, M., and Watanabe, H. 1997. NMDA antagonists potentiate scopolamine-induced amnesic effect. Behav. Brain Res. 83: 225-228.

Linster, C., Garcia, P.A., Hasselmo, M.E., and Baxter, M.G. 2001. Selective loss of cholinergic neurons projecting to the olfactory system increases perceptual generalization between similar, but not dissimilar, odorants. Behav. Neurosci. 115: 826-833.

Miasnikov, A.A., McLin III, D., and Weinberger, N.M. 2001. Muscarinic dependence of nucleus basalis induced conditioned receptive field plasticity. Neuroreport 12: $1537-1542$.

Miranda, M.I. and Bermudez-Rattoni, F. 1999. Reversible inactivation of the nucleus basalis magnocellularis induces disruption of cortical acetylcholine release and acquisition, but not retrieval, of aversive memories. Proc. Natl. Acad. Sci. 96: 6478-6482.

Miranda, M.I. and Bermudez-Rattoni, F. 2007. Cholinergic activity in the insular cortex is necessary for acquisition and consolidation of contextual memory. Neurobiol. Learn. Mem. 87: 343-351.

Miranda, M.I., Ramirez-Lugo, L., and Bermudez-Rattoni, F. 2000. Cortical cholinergic activity is related to the novelty of the stimulus. Brain Res. 882: 230-235.

Miranda, M.I., Ferreira, G., Ramirez-Lugo, L., and Bermudez-Rattoni, F. 2003. Role of cholinergic system on the construction of memories: Taste memory encoding. Neurobiol. Learn. Mem. 80: 211-222.

Monteiro Moreira, K., Lima Ferreira, T., Vecchio Fornari, R., Perez Figueredo, L.Z., and Menezes Oliveira, M.G. 2005. Interaction between M1-muscarinic and glutamatergic NMDA receptors on an inhibitory avoidance task. Brain Res. Bull. 67: 504-508.

Neafsey, E.J., Hurley-Gius, K.M., and Arvanitis, D. 1986. The topographical organization of neurons in the rat medial frontal, insular and olfactory cortex projecting to the solitary nucleus, olfactory bulb, periaqueductal gray and superior colliculus. Brain Res. 377: $561-570$

Ohno, M. and Watanabe, S. 1996. Interactive processing between glutamatergic and cholinergic systems involved in inhibitory avoidance learning of rats. Eur. J. Pharmacol. 312: 145-147.

Ongur, D. and Price, J.L. 2000. The organization of networks within the orbital and medial prefrontal cortex of rats, monkeys and humans. Cereb. Cortex 10: 206-219.

Orsetti, M., Casamenti, F., and Pepeu, G. 1996. Enhanced acetylcholine release in the hippocampus and cortex during acquisition of an operant behavior. Brain Res. 724: 89-96.

Paxinos, G. and Watson, C. 1997. The rat brain in stereotaxic coordinates. Elsevier Academic Press, Amsterdam, The Netherlands.

Power, A.E., Thal, L.J., and McGaugh, J.L. 2002. Lesions of the nucleus basalis magnocellularis induced by 192 IgG-saporin block memory enhancement with posttraining norepinephrine in the basolateral amygdala. Proc. Natl. Acad. Sci. 99: 2315-2319.

Power, A.E., Vazdarjanova, A., and McGaugh, J.L. 2003. Muscarinic cholinergic influences in memory consolidation. Neurobiol. Learn. Mem. 80: 178-193.

Quirarte, G.L., Cruz-Morales, S.E., Cepeda, A., Garcia-Montanez, M., Roldan-Roldan, G., and Prado-Alcala, R.A. 1994. Effects of centra muscarinic blockade on passive avoidance: Anterograde amnesia, state dependency, or both? Behav. Neural Biol. 62: 15-20.

Quiroz-Padilla, M.F., Guillazo-Blanch, G., Vale-Martínez, A., and Marti-Nicolovius, M. 2006. Excitotoxic lesions of the parafascicular nucleus produce deficits in a socially transmitted food preference. Neurobiol. Learn. Mem. 86: 256-263.

Quiroz-Padilla, M.F., Guillazo-Blanch, G., Vale-Martínez, A., Torras-Garcia, M., and Marti-Nicolovius, M. 2007. Effects of parafascicular excitotoxic lesions on two-way active avoidance and odor discrimination. Neurobiol. Learn. Mem. 88: 198-207.

Ragozzino, M.E. 2000. The contribution of cholinergic and dopaminergic afferents in the rat prefrontal cortex to learning, memory and attention. Psychobiology 28: 238-247.

Ragozzino, M.E. and Kesner, R.P. 1998. The effects of muscarinic cholinergic receptor blockade in the rat anterior cingulate and Prelimbic/Infralimbic cortices on spatial working memory. Neurobiol. Learn. Mem. 69: 241-257.

Ragozzino, M.E., Detrick, S., and Kesner, R.P. 1999. Involvement of the prelimbic-infralimbic areas of the rodent prefrontal cortex in behavioral flexibility for place and response learning. J. Neurosci. 19: $4585-4594$

Ragozzino, M.E., Kim, J., Hassert, D., Minniti, N., and Kiang, C. 2003. The contribution of the rat prelimbic-infralimbic areas to different forms of task switching. Behav. Neurosci. 117: 1054-1065. 
Ramirez-Lugo, L., Miranda, M.I., Escobar, M.L., Espinosa, E., and Bermudez-Rattoni, F. 2003. The role of cortical cholinergic pre- and post-synaptic receptors in taste memory formation. Neurobiol. Learn. Mem. 79: 184-193.

Ravel, N., Elaagouby, A., and Gervais, R. 1994. Scopolamine injection into the olfactory bulb impairs short-term olfactory memory in rats. Behav. Neurosci. 108: 317-324.

Riekkinen Jr., P., Kuitunen, J., and Riekkinen, M. 1995. Effects of scopolamine infusions into the anterior and posterior cingulate on passive avoidance and water maze navigation. Brain Res. 685: 46-54.

Robbins, T.W. 2005. Chemistry of the mind: Neurochemical modulation of prefrontal cortical function. J. Comp. Neurol. 493: 140-146.

Robbins, T.W., Granon, S., Muir, J.L., Durantou, F., Harrison, A., and Everitt, B.J. 1998. Neural systems underlying arousal and attention. Implications for drug abuse. Ann. N. Y. Acad. Sci. 846: 222-237.

Roman, F.S., Simonetto, I., and Soumireu-Mourat, B. 1993. Learning and memory of odor-reward association: Selective impairment following horizontal diagonal band lesions. Behav. Neurosci. 107: 72-81.

Saar, D., Grossman, Y., and Barkai, E. 2001. Long-lasting cholinergic modulation underlies rule learning in rats. J. Neurosci.

21: $1385-1392$.

Sara, S.J., Roullet, P., and Przybyslawski, J. 1999. Consolidation of memory for odor-reward association: $\beta$-adrenergic receptor involvement in the late phase. Learn. Mem. 6: 88-96.

Toumane, A., Durkin, T., Marighetto, A., Galey, D., and Jaffard, R. 1988. Differential hippocampal and cortical cholinergic activation during the acquisition, retention, reversal and extinction of a spatial discrimination in an 8-arm radial maze by mice. Behav. Brain Res. 30: $225-234$.

Torras-Garcia, M., Lelong, J., Tronel, S., and Sara, S.J. 2005. Reconsolidation after remembering an odor-reward association requires NMDA receptors. Learn. Mem. 12: 18-22.

Tronel, S. and Sara, S.J. 2002. Mapping of olfactory memory circuits: Region-specific c-fos activation after odor-reward associative learning or after its retrieval. Learn. Mem. 9: 105-111.

Tronel, S. and Sara, S.J. 2003. Blockade of NMDA receptors in prelimbic cortex induces an enduring amnesia for odor-reward associative learning. J. Neurosci. 23: 5472-5476.

Tronel, S., Feenstra, M.G., and Sara, S.J. 2004. Noradrenergic action in prefrontal cortex in the late stage of memory consolidation. Learn. Mem. 11: 453-458.

Vale-Martínez, A., Baxter, M.G., and Eichenbaum, H. 2002. Selective lesions of basal forebrain cholinergic neurons produce anterograde and retrograde deficits in a social transmission of food preference task in rats. Eur. J. Neurosci. 16: 983-998.

Wilson, D.A., Fletcher, M.L., and Sullivan, R.M. 2004. Acetylcholine and olfactory perceptual learning. Learn. Mem. 11: 28-34.

Wrenn, C.C., Harris, A.P., Saavedra, M.C., and Crawley, J.N. 2003. Social transmission of food preference in mice: Methodology and application to galanin-overexpressing transgenic mice. Behav. Neurosci. 117: 21-31.

Received April 3, 2007; accepted in revised form July 9, 2007. 


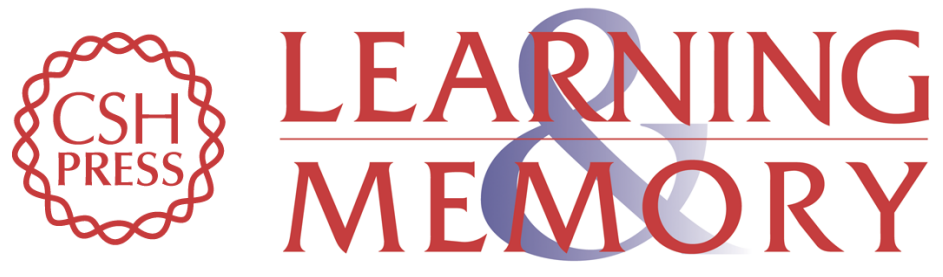

\section{Differential effects of muscarinic receptor blockade in prelimbic cortex on acquisition and memory formation of an odor-reward task}

Anna Carballo-Márquez, Anna Vale-Martínez, Gemma Guillazo-Blanch, et al.

Learn. Mem. 2007, 14:

Access the most recent version at doi:10.1101//m.597507

References This article cites 58 articles, 13 of which can be accessed free at:

http://learnmem.cshlp.org/content/14/9/616.full.html\#ref-list-1

License

Email Alerting

Receive free email alerts when new articles cite this article - sign up in the box at the Service top right corner of the article or click here. 